\title{
Pat1 activates late steps in mRNA decay by multiple mechanisms
}

\author{
Joseph H. Lobel ${ }^{a, b}$, Ryan W. Tibble ${ }^{a, b}$, and John D. Gross ${ }^{b, 1}$ \\ ${ }^{a}$ Chemistry and Chemical Biology Graduate Program, University of California, San Francisco, CA 94158; and ${ }^{b}$ Department of Pharmaceutical Chemistry, \\ University of California, San Francisco, CA 94158
}

Edited by Roy Parker, University of Colorado Boulder, Boulder, CO, and approved October 10, 2019 (received for review April 3, 2019)

\begin{abstract}
Pat1 is a hub for mRNA metabolism, acting in pre-mRNA splicing, translation repression, and mRNA decay. A critical step in all $5^{\prime}-3^{\prime}$ mRNA decay pathways is removal of the $5^{\prime}$ cap structure, which precedes and permits digestion of the RNA body by conserved exonucleases. During bulk 5'-3' decay, the Pat1/Lsm1-7 complex engages mRNA at the $3^{\prime}$ end and promotes hydrolysis of the cap structure by Dcp1/Dcp2 at the $5^{\prime}$ end through an unknown mechanism. We reconstitute Pat1 with $5^{\prime}$ and $3^{\prime}$ decay factors and show how it activates multiple steps in late mRNA decay. First, we find that Pat1 stabilizes binding of the Lsm1-7 complex to RNA using two conserved short-linear interaction motifs. Second, Pat1 directly activates decapping by binding elements in the disordered C-terminal extension of Dcp2, alleviating autoinhibition and promoting substrate binding. Our results uncover the molecular mechanism of how separate domains of Pat1 coordinate the assembly and activation of a decapping messenger ribonucleoprotein (mRNP) that promotes 5'-3' mRNA degradation.
\end{abstract}

mRNA decay | decapping | Pat1 | Dcp2 | short-linear interaction motifs

$\mathbf{P}$ roper degradation of mRNA transcripts shapes the timing and quantity of gene expression to regulate a diverse array of cellular processes $(1,2)$. Cleavage of the $5^{\prime} \mathrm{m}^{7} \mathrm{G}$ cap (decapping) removes the mRNA from the translating pool and exposes a free monophosphate which leads to rapid degradation by the $5^{\prime}-3^{\prime}$ exonuclease $\operatorname{Xrn} 1(2,3)$. Decapping is a critical process for establishing $5^{\prime}-3^{\prime}$ mRNA degradation and is found in bulk mRNA decay, clearance of maternal transcripts, quality control pathways, and miRNA mediated decay (4-7). The Dcp1/Dcp2 holoenzyme is a conserved NUDIX hydrolase that cleaves the $5^{\prime} \mathrm{m}^{7} \mathrm{G}$ cap and is targeted to specific mRNAs by cofactors $(1,8-$ 13). These cofactors regulate the activity of Dcp1/Dcp2 by either binding the catalytic core of the enzyme or binding helical leucine motifs (HLMs) in the disordered C terminus of Dcp2, which contains additional cis-regulatory elements that inhibit decapping (14-17). Thus, a dense network of protein interactions has evolved to coordinate decapping of specific mRNA targets (18).

Bulk $5^{\prime}-3^{\prime}$ mRNA degradation begins with trimming of the $3^{\prime}$ poly(A) tail, which may be followed by the addition of 1-3 uridines by terminal uridyl transferases in higher order eukaryotes $(19,20)$. Deadenylation results in loss of translation initiation factors and permits the assembly of a decapping mRNP (21). Pat1 and Lsm1-7 work as a complex that assembles at the $3^{\prime}$ end of a mRNA to, subsequently, promote decapping by Dcp1/Dcp2 (21-26). Structural and biochemical studies have demonstrated that Pat1 and Lsm1-7 form a heterooctameric complex that engages transcripts containing oligoA tails that result from deadenylation (27-30). Pat1 and Lsm1-7 are functionally linked as they bind the $3^{\prime}$ end of mRNA, and deletion of either Pat1 or Lsm1 causes an increase in steady-state levels of numerous overlapping transcripts $(12,30)$. In addition, deletion of Pat1 or Lsm1 results in an accumulation of deadenylated capped mRNA intermediates, suggesting a block in decapping $(21,22,31)$. Finally, Pat1 forms a core component of processing bodies (Pbodies), and can recruit interacting partners to these cytoplasmic foci, which may function in mRNA storage or decay (32-
34). A critical and unresolved question is how Pat1 coordinates assembly of the Lsm1-7 complex on the mRNA $3^{\prime}$ end with decapping at the $5^{\prime}$ end.

Pat 1 is conserved from yeast to humans and contains 3 domains that interact with distinct decay factors. The disordered $\mathrm{N}$-terminal region of Pat1 contains a conserved motif that interacts with the DEAD-box ATPase Dhh1, but is generally dispensable for cell growth and normal mRNA turnover $(35,36)$ The $\alpha-\alpha$ superhelical C-terminal domain (PatC) interacts with Lsm1-7, which is required for bulk $5^{\prime}-3^{\prime}$ mRNA degradation in vivo $(27,28,37-39)$. Removal of the unstructured middle domain severely attenuates decapping and $5^{\prime}-3^{\prime}$ degradation, although the mechanism remains poorly understood $(36,40)$. The conservation of the middle and C-terminal domains from yeast to humans suggests they are critical for decapping and proper mRNA decay, but how these domains interact with and activate mRNA decay factors remains unknown.

Here, we determine how Pat1 interacts with and activates both the Lsm1-7 complex and the Dcp1/Dcp2 enzyme complex from fission yeast Schizosaccaromyces pombe (Sp) using recombinant purified proteins, which recapitulate the regulation of decapping observed in budding yeast $(16,17)$. We show that Pat1 promotes RNA binding of the Lsm1-7 complex using two shortlinear motifs from its middle domain that make physical interactions with the Lsm1-7 ring and enhance its interaction with RNA. Pat1 activates decapping using its middle domain and structured $\mathrm{C}$ terminus, which directly binds conserved HLMs in Dcp2, to alleviate autoinhibition and promote substrate binding. Our results reveal how Pat1 nucleates assembly of a decapping mRNP and uses distinct domains to activate decay factors at

\section{Significance}

In yeast, the majority of mRNAs is degraded in a $5^{\prime}-3^{\prime}$ direction. This pathway requires the assembly of conserved protein complexes on the $5^{\prime}$ and $3^{\prime}$ termini of the mRNA after its poly(A) tail is removed. These decay complexes are poorly active in the absence of the Pat1 protein. We show how Pat1 enhances binding of the $3^{\prime}$ complex to RNA and activates the 5 decapping complex using a combination of disordered and structured domains to promote distinct steps in mRNA decay. By activating cofactors, Pat1 may be able to regulate multiple aspects of mRNA metabolism.

Author contributions: J.H.L. and J.D.G. designed research; J.H.L. and R.W.T. performed research; J.H.L. analyzed data; and J.H.L. and J.D.G. wrote the paper.

The authors declare no competing interest.

This article is a PNAS Direct Submission.

Published under the PNAS license.

Data deposition: Plots and fits were generated using in house scripts written in $\mathrm{R}$, which are available at https://github.com/jhlobel/FP-fitting.

${ }^{1}$ To whom correspondence may be addressed. Email: jdgross@cgl.ucsf.edu.

This article contains supporting information online at www.pnas.org/lookup/suppl/doi:10. 1073/pnas.1905455116/-/DCSupplemental.

First published November 5, 2019. 
both the $3^{\prime}$ and the $5^{\prime}$ ends of a transcript to promote mRNA degradation.

\section{Results}

Pat1 Makes Bipartite Interactions with Lsm1-7 to Enhance RNA Binding. Previous studies of Pat1 and Lsm1-7 purified from budding yeast indicate Pat1 is necessary to promote high affinity interactions with Lsm1-7 and RNA, and that the middle and Cterminal domains of Pat1 are sufficient to complement growth defects in strains where Pat1 is deleted $(36,37)$. To understand the domains of Pat1 involved in mRNA turnover in $S p$, we constructed strains harboring different domain deletions of Pat1(Fig. 1A). Both FL-Pat1 and PatMC (residues 296-754) were sufficient to complement the growth defect in the $\Delta$ Pat1 strain. Neither the middle nor the C-terminal domain alone, however, could rescue growth, which was not due to a protein expression defect (Fig. $1 B$ and $C$ ). This suggests that, like budding yeast, both the middle and the C-terminal domains of Pat1 are required for normal mRNA turnover in $S p$.

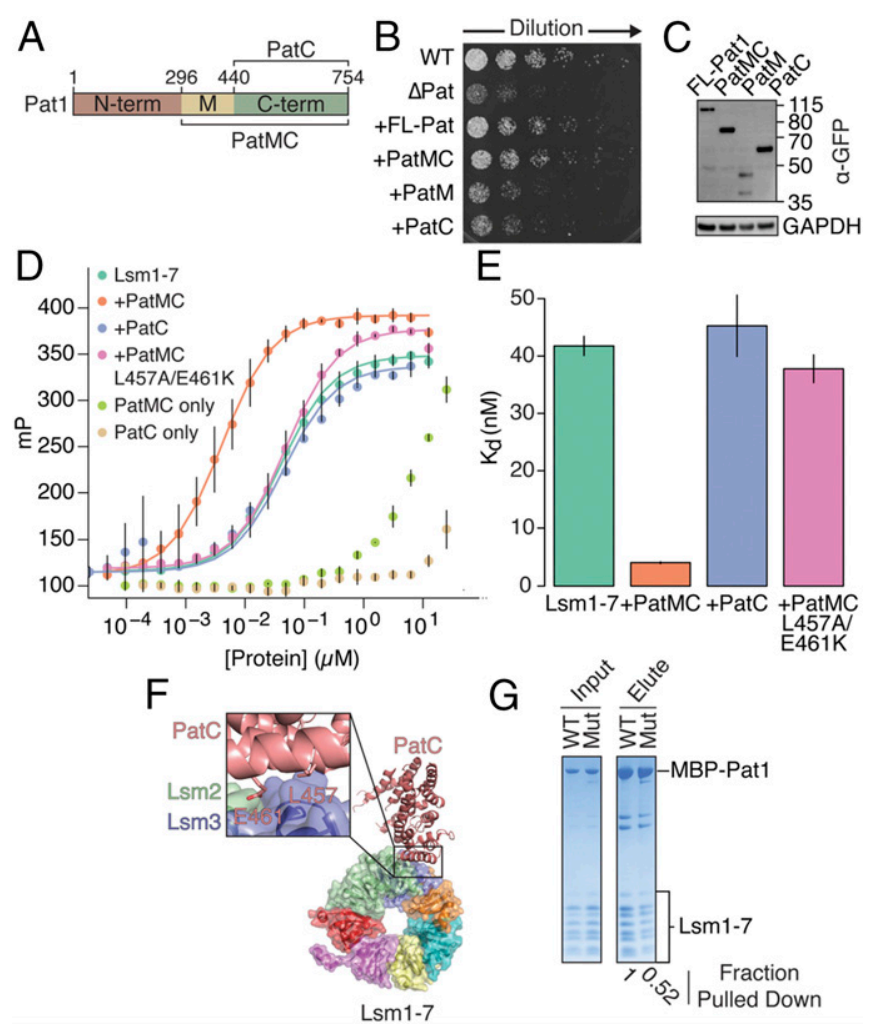

Fig. 1. Pat1 makes a bipartite interaction with Lsm1-7 to enhance RNA binding. (A) Schematic of Pat1 domains. (B) Plate growth assay of $S p$ at $30^{\circ} \mathrm{C}$ for $\sim 1.5$ days with 4-fold dilutions from $\mathrm{OD}_{600}=0.115$ on YES media. FL-Pat 1 refers to full-length Pat1. All proteins were expressed as C-terminal superfolderGFP fusions under control of the endogenous promoter and with the Ura4 3' UTR. (C) Western blot for expression of different Pat1 constructs detected with a GPF antibody and GAPDH as a loading control. Molecular weights in $\mathrm{kDa}$ are shown on the right. $(D)$ Lsm1-7 binding 5' FAM-rA15 RNA (Lsm1-7, $n=6$; +PatMC, $n=4$, +PatC; and +L457A/E461K, $n=2$ ) monitored by fluorescence polarization. All Pat1 constructs are $\mathrm{N}$-terminal $\mathrm{His}_{6} \mathrm{MBP}$ (maltose binding protein) fusion proteins. $(E)$ Equilibrium dissociation constants determined from fits in $D$ with standard deviation. $(F)$ Crystal structure of PatC interaction with Lsm1-7 (PDB 4C8Q) with magnification of the PatC region contacting $L s m 2$ and Lsm3. Residues are numbered according to fission yeast and are conserved. (G) MBP pull-down assay using $10 \mu \mathrm{M}$ Lsm1-7 with stoichiometric amounts of MBP-Pat1 construct as input. Mut refers to the SpPatMC L457A/E461K variant.
To better understand how individual domains of Pat1 function in a complex with Lsm1-7, we purified MBP fusions of different fission yeast Pat1 constructs and queried if they could enhance RNA binding of the Lsm1-7 complex. We used fluorescence polarization with a rA15 probe (termed oligoA), which mimics a deadenylated tail, to measure RNA binding and examined which regions of Pat1 could enhance the association of Lsm1-7 with RNA. The addition of MBP-PatMC enhanced the RNA binding of Lsm1-7, while PatC showed no effect (Fig. $1 D$ and $E$ ). Importantly, PatMC alone bound oligoA RNA weakly, indicating the enhancement of RNA binding was due to the enhancement of Lsm1-7 RNA binding. This effect was specific to oligoA as Pat1 did not enhance binding to a U15 probe (SI Appendix, Fig. S1 $A-C$ ). To test if PatC's failure to enhance RNA binding of Lsm1-7 was simply due to an inability to form a PatC/Lsm1-7 complex, we incubated PatC and Lsm1-7 together and were unable to observe a stable complex by analytical size exclusion chromatography (SI Appendix, Fig. S2 $A$ and $B$ ). This indicates that the PatC/Lsm1-7 interaction is likely weak and that the middle domain of Pat1 is required to enhance RNA binding of Lsm1-7, in part, by promoting the formation a stable heterooctameric complex.

Previous crystal structures have identified conserved residues on PatC that contact the Lsm $2 / 3$ subunits and, when mutated, lead to loss of complex formation and defects in mRNA degradation in budding yeast $($ Fig. $1 F)(27,28)$. Introducing these mutations into SpPatMC (L457A/E461K) minimally affected its association with Lsm1-7 as shown by pull-down analysis but completely abrogated its ability to enhance Lsm1-7 RNA binding (Fig. $1 D, E$, and $G$ ). This suggests that PatC must make specific interactions with Lsm $2 / 3$ to promote RNA binding and that the middle domain of Pat1 is required to form a stable complex with Lsm1-7. Furthermore, the inability of PatMC L457A/E461K to promote Lsm1-7 RNA binding suggests the middle domain is necessary but not sufficient to enhance function of the complex. We conclude that interactions of the middle and C-terminal domains of Pat1 with the Lsm1-7 ring are required to enhance RNA binding.

Short-Linear Motifs in the Middle Domain of Pat1 Bind Lsm1-7 and Enhance RNA Binding. The middle domain of Pat1 could enhance RNA binding of Lsm1-7 by directly binding RNA, promoting a stable complex formation with Lsm1-7, or through both mechanisms. We first sought to identify which domain of Pat1 could associate with Lsm1-7. A construct containing most of the middle domain of Pat1, specifically residues 296-431, was necessary and sufficient to interact with Lsm1-7, while PatC was unable to form a stable complex by pull-down analysis (Fig. 2). This suggests the middle domain of Pat1 is responsible for promoting a stable complex with Lsm1-7.

We wanted to distinguish if the middle domain was only involved in protein-protein interactions or had additional roles in promoting RNA binding. To test these possibilities, we purified a series of N-terminal truncations of Pat 1 and surveyed their ability to associate with Lsm1-7 by pull-down assays (Fig. $2 A$ and $B$, truncations 1-4). Deletion of residues 296-431 abolished the Pat1/Lsm1-7 interaction, indicating that these residues are involved in associating with Lsm1-7 (truncation 3). This identifies residues in the middle domain of Pat1 that are required to form a stable complex with Lsm1-7.

Because we identified two N-terminal truncations of Pat 1 that retained the ability to interact with Lsm1-7 to a similar extent, we tested if these could enhance RNA binding of the PatMC/Lsm1-7 complex (Fig. $2 A$ and $B$, truncations 1 and 2 ). If the sole function of the middle domain is to promote association with Lsm1-7, then all N-terminal truncations that interact with Lsm1-7 should enhance RNA binding. Contrary to this expectation, we found Pat1 317-754 was unable to stimulate RNA binding (Fig. $2 C$ and $D$ ). This suggests that PatMC contains a region that is dispensable for PatMC/Lsm1-7 complex formation but essential for enhancing 
A
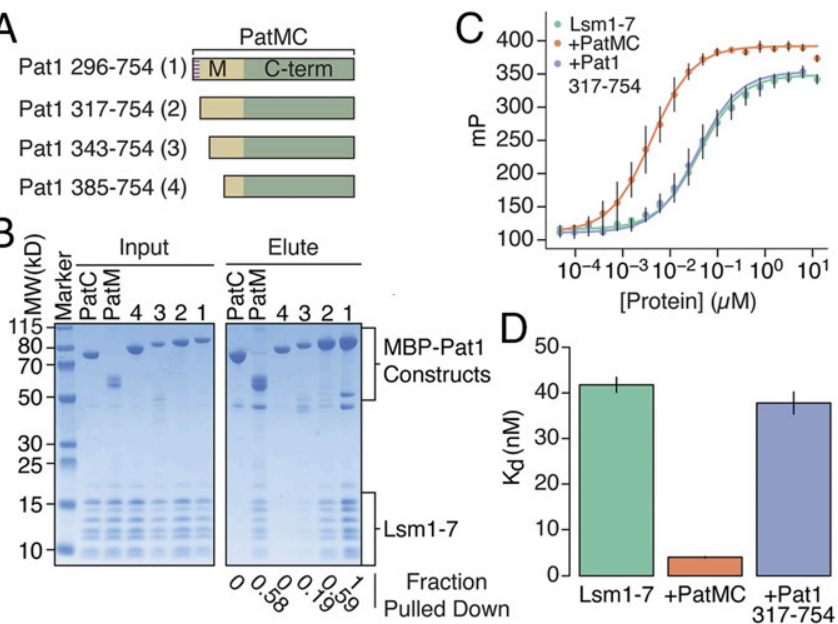

[Protein] $(\mu \mathrm{M})$

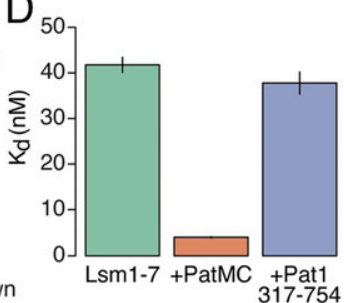

E

S. OC.

S. cryophilus (308-328) L L E K R K K LER D HM LMAQ

S. japonicus (269-289) QY LKQQ HV EK T RQL I GRCAC
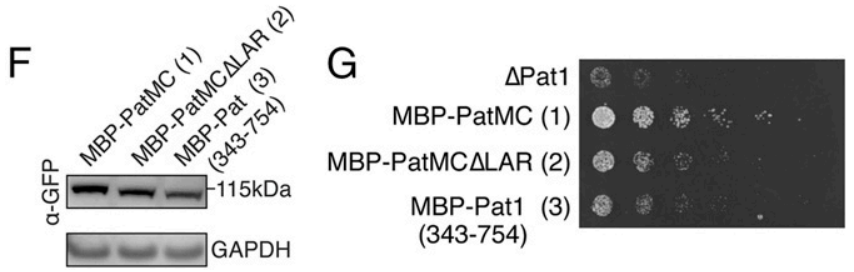

Fig. 2. Separate regions of the middle domain of Pat1 interact with and enhance RNA binding by Lsm1-7. (A) Schematic of different MBP-Pat1 constructs used in pull-down and binding assays. The Lsm activation region (LAR) is shown in stripes. (B) Pull-down of $10 \mu \mathrm{M}$ Lsm1-7 with stoichiometric amounts of the specified MBP-Pat1 construct. The numbers refer to the schematic in $A$. (C) Fluorescence polarization of $5^{\prime}$ FAM-rA15 binding to reconstituted MBP-Pat1:Lsm1-7 complexes with SD (Lsm1-7, $n=6 ;+$ MBPPatMC, $n=4 ;+$ MBP Pat1 317-754, $n=4$ ). All conditions contain stoichiometric amounts of Pat1/Lsm1-7. (D) Dissociation constants of determined from the fit to $C$ with error bars representing SD. (E) Sequence alignment of the LAR in different Schizosaccharomyces species. $(F)$ Western blot for the expression of different Pat 1 constructs detected with a GPF antibody and GAPDH as a loading control. (G) Plate growth assay of $S p$ at $30^{\circ} \mathrm{C}$ for $\sim 1.5 \mathrm{~d}$ with 4 -fold dilutions from $\mathrm{OD}_{600}=0.115$ on YES media. All proteins were expressed as $\mathrm{N}$ terminal MBP fusions to aid expression and C-terminal superfolderGFP fusions under control of the endogenous promoter and with the Ura4 3' UTR.

RNA binding. The binding isotherm for Pat1 317-754 is identical to the Lsm1-7/PatMC L457A/E461K mutant complex, which can also interact with Lsm1-7 but fails to enhance RNA binding (Fig. $1 B, C$, and $E$ ). We, therefore, reason that residues 296-316 of Pat1 must be involved in stabilizing a productive complex that can bind RNA with high affinity, which we term the 'Lsm Activating Region' (LAR). The LAR is highly conserved in the Schizosaccharomyces genus, which is characteristic of short-linear motifs that can be rapidly transferred between proteins during evolution (Fig. 2E) (18). To understand the importance of the LAR for Pat1's function in vivo, we tested the ability of PatMC truncations to rescue cell growth. PatMC truncations were fused to a N-terminal MBP to permit expression (Fig. $2 F$ ). Deletion of the LAR resulted in a slow growth phenotype compared to PatMC alone. Additional truncation of Pat1 did not further reduce cell growth (Fig. 2G). Therefore, we conclude that the LAR of Pat1 is important for function in fission yeast. Metazoans have gained additional residues in this region, although we envision the LAR is still required for Lsm1-7 activation in higher order eukaryotes (SI Appendix, Fig. S3).
Our pull-down analysis and functional studies suggest that residues 317-342 in the middle domain of Pat1 are important for promoting a stable PatMC/Lsm1-7 complex. Sequence alignments reveal this region is conserved in Pat1 from yeast to humans (Fig. $3 A$ ). To determine if this motif is necessary for the interaction of Pat1 with Lsm1-7, we constructed a series of internal PatMC deletions by replacing intervening residues with a flexible (GS) linker (Fig. 3B). Because appending the conserved motif containing amino acids $317-342$ of the middle domain to PatC stabilized the interaction with Lsm1-7 (Fig. 3C), we refer to this region of Pat1 as the 'Lsm Binding Motif' (LBM). In contrast, deletion of the LAR was dispensable for Pat1/Lsm1-7 interactions. While we cannot exclude that additional regions of Pat1 help stabilize the Pat1/Lsm1-7 complex, these data suggest the LBM helps mediate the protein-protein interaction with the Lsm1-7 ring. We conclude the middle domain of Pat1 contains two short-linear motifs that may function separately in stabilizing the PatMC/Lsm1-7 complex and a conformation that is productive for RNA binding.

The Middle and C-Terminal Domains of Pat1 Activate Dcp1/Dcp2. Numerous genetic experiments have demonstrated that the middle and C-terminal domains of Pat 1 are required to enhance decapping in vivo, but the molecular mechanism has remained unclear $(28,36)$. Dcp2 contains a catalytic NUDIX hydrolase domain that cleaves the $\mathrm{m} 7 \mathrm{G}$ cap as well as a disordered C-terminal extension that is replete with regulatory elements, such as inhibitory motifs and protein-protein interaction motifs. While we could not purify full-length Dcp2, a C-terminally extended Dcp2 containing up to 261 additional amino acids recapitulates the regulatory elements in the full-length C-terminal extension (Fig. 4A) $(16,17)$. The $\mathrm{C}$-terminal domain of budding yeast Pat1 was crystallized with a HLM of Dcp2, suggesting a mechanism by which Pat1 could promote decapping (41). Accordingly, we tested the ability of Pat1 to activate the decapping holoenzyme Dcp1/Dcp2 in vitro using a C-terminally extended Dcp2 that contains two HLMs (Dcp1/Dcp2 1-318, termed Dcp1/Dcp2 ${ }_{\mathrm{HLM} 1 / 2}$ ) (Fig. 4A) (41, 42). We chose this Dcp1/Dcp2 construct for initial studies to examine how Pat1-binding Dep2 could function independently of inhibitory elements in the C-terminal extension of Dcp2. PatMC was able to enhance the activity of Dcp1/Dcp2 ${ }_{\mathrm{HLM} 1 / 2}$, while PatC had little effect on activity (Fig. 4B). This suggests that both the middle and the C-terminal domain of Pat1 cooperate to directly activate decapping.

Although PatC was unable to stimulate decapping on its own, we next asked if it could directly associate with HLMs of Dcp2 using ${ }^{15} \mathrm{~N}$ HSQC NMR spectroscopy. We purified ${ }^{15} \mathrm{~N}$ labeled Dcp2 containing a single HLM (Dcp2 1-266, termed Dcp2-HLM1,

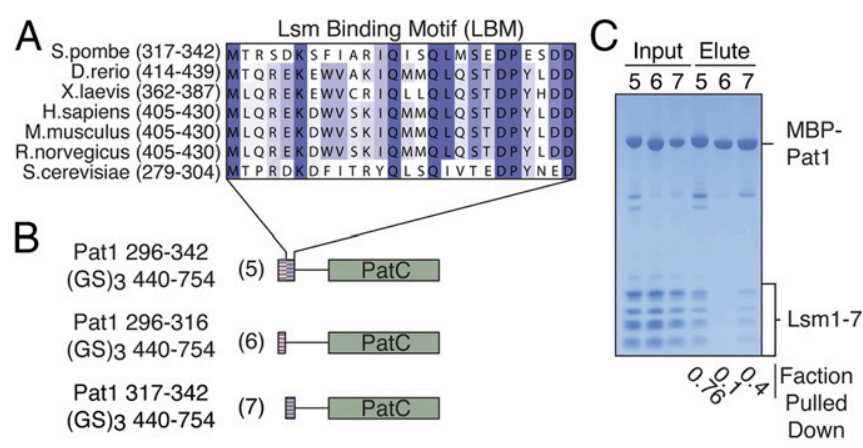

Fig. 3. A short-linear motif in the middle domain of Pat1 mediates proteinprotein interaction with Lsm1-7. (A) Conservation of the Lsm-binding motif (LBM) in the Pat1 middle domain between different species. (B) Schematic of constructs used. Residues were deleted and replaced with a flexible 6 amino acid (GS) 3 linker. The LAR and LBM are shown in red or purple stripes, respectively. (C) Pull-down of $10 \mu \mathrm{M}$ Lsm1-7 with stoichiometric amounts of MBP-PatMC internal deletions. The numbers refer to the schematic in $A$. 


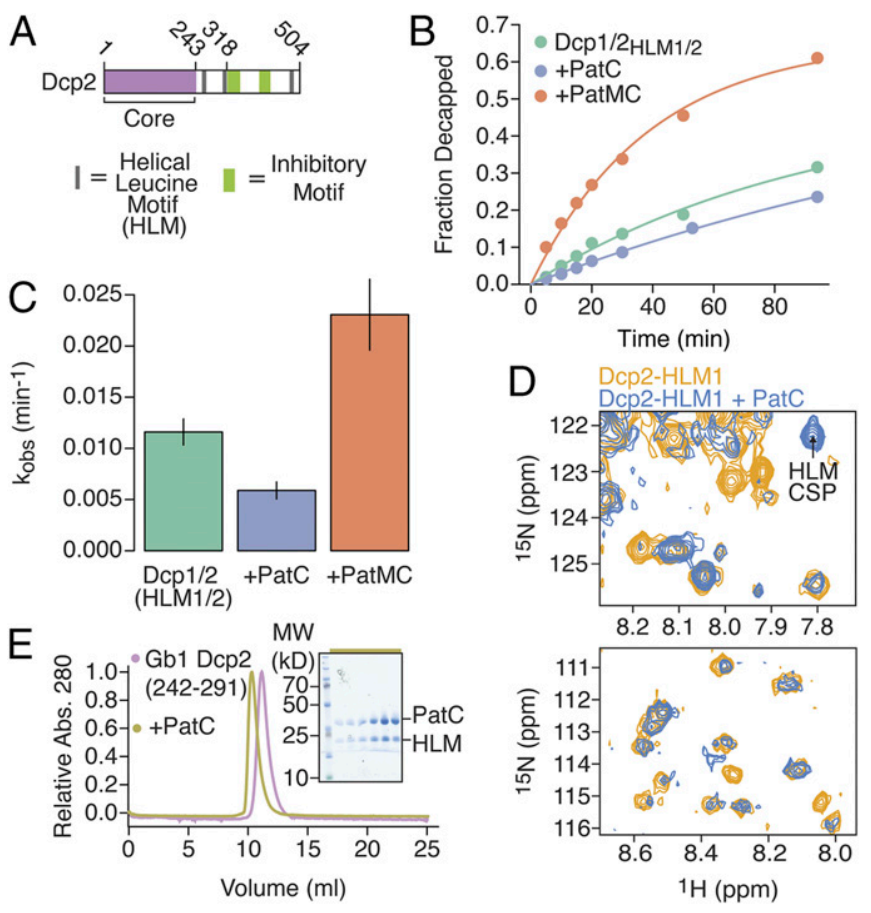

Fig. 4. Pat1 directly binds and activates C-terminally extended Dcp2 constructs. $(A)$ Schematic of domains of Dcp2. The structured core that hydrolyzes cap is shown in purple. The gray boxes represent HLMs, and the green boxes represent inhibitory elements. $(B)$ Representative decapping of an RNA with $150 \mathrm{nM} \mathrm{Dcp} 1 / \mathrm{Dcp} 2_{\mathrm{HLM} 1 / 2}$ and $10 \mu \mathrm{M}$ of MBP-tagged Pat 1 construct. (C) Rates determined from $B$ displayed with SD $(n=2)$. (D) Magnified views of regions of interest in ${ }^{1} \mathrm{H}-{ }^{15} \mathrm{~N}$ HSQC of $100 \mu \mathrm{M}$ DCp2 $2_{\mathrm{HLM} 1}$ with or without $150 \mu \mathrm{M}$ PatC. The full spectrum is in SI Appendix, Fig. S1. (E) Analytical size exclusion chromatography of $35 \mu \mathrm{M} \mathrm{H} \mathrm{H}_{6}-\mathrm{GB} 1-\mathrm{Dcp} 2$ 242-291 alone (pink) or with a stoichiometric amount of PatC (gold). Inset is gel corresponding to the peak in the gold trace. The HLM sequence is residues 257-264 of Dcp2.

Fig. 4A), and determined if any chemical shift perturbations (CSPs) occurred in the presence of PatC. Upon addition of PatC, we observed losses and reappearances of resonances in Dcp2HLM1 but not in the Dcp2 catalytic core alone (Dcp2 1-243, termed Dcp2 $2_{\text {Core }}$ ) (Fig. $4 D$ and SI Appendix, Fig. S4). This indicates that PatC directly binds HLMs in Dcp2. Furthermore, HLM-1 of Dcp2 was sufficient for binding PatC, as assayed by NMR CSP analysis and copurification by size-exclusion chromatography (Fig. $4 E$ and SI Appendix, Fig. S5 $A-D$ ). We conclude that fission yeast PatC is sufficient to interact with Dcp2 by binding HLMs, but both the middle and the C-terminal domains are required to activate decapping (41).

To gain insight into how Pat1 activates decapping by Dcp1/ Dcp2 in the absence of inhibitory elements, we determined the kinetic parameters of Dcp1/Dcp2 ${ }_{\mathrm{HLM} 1 / 2}$ either alone or in the presence of MBP-PatMC using a single-turnover decapping assay (Fig. 5A). We used saturating PatMC in all experiments to ensure complete formation of a PatMC/Dcp1/Dcp2 complex at all measured concentrations. While we observed minimal effects on $\mathrm{k}_{\max }$, we saw an $\sim 3$-fold reduction in $\mathrm{K}_{\mathrm{m}}$ (Fig. $5 E$ and $F$ ). This suggests that PatMC can bind to HLMs in Dcp2 to enhance substrate binding of the decapping complex.

While SpPatC had no effect on decapping in vitro, we hypothesized that, like the Lsm1-7 complex, both the middle domain and the C-terminal domains of Pat1 may cooperate to enhance the activity of Dcp1/Dcp2. Studies of human PatC identified a basic patch that is required for mRNA decapping and decay (38). Modeling of $S p$ PatC onto existing crystal structures reveals a similar basic patch to that identified in humans, which we reasoned may be involved in activating Dcp2 (Fig. $5 B$ ). Mutation of these 4 basic residues to alanine (K453A/K454A/K530A/ $\mathrm{R} 533 \mathrm{~A}$, referred to as a $4 \times$ mutant) in SpPatMC abolished its ability to activate Dcp1/Dcp2 (Fig. 5C). Because the middle domain and residues on the C-terminal domain of Pat1 are both required to activate decapping, these domains may form a bipartite RNA-binding surface that can recruit Dcp1/Dcp2 to the substrate. This suggests that PatC can bind HLMs and cooperate with the middle domain of Pat1 to increase the affinity of Dcp1/ Dcp2 for substrate RNA.

In addition to HLMs, the C-terminal region of Dcp2 contains inhibitory elements that promote nonproductive binding of Dcp1/Dcp2 to the substrate to limit catalysis (Fig. 4A). Other HLM-binding proteins, such as Edc3, have been shown to relieve autoinhibition (17). To interrogate how PatMC activates an autoinhibited Dcp1/Dcp2, we determined the kinetic parameters of an extended Dcp1/Dcp2 construct that contains both inhibitory elements and HLMs (Dcp1/Dcp2 1-504, termed Dcp1/Dcp2 Ext) either alone or with saturating amounts of PatMC. Unlike the Dcp1/Dcp2 ${ }_{\mathrm{HLM} 1 / 2}$, PatMC activated the catalytic step of decapping,
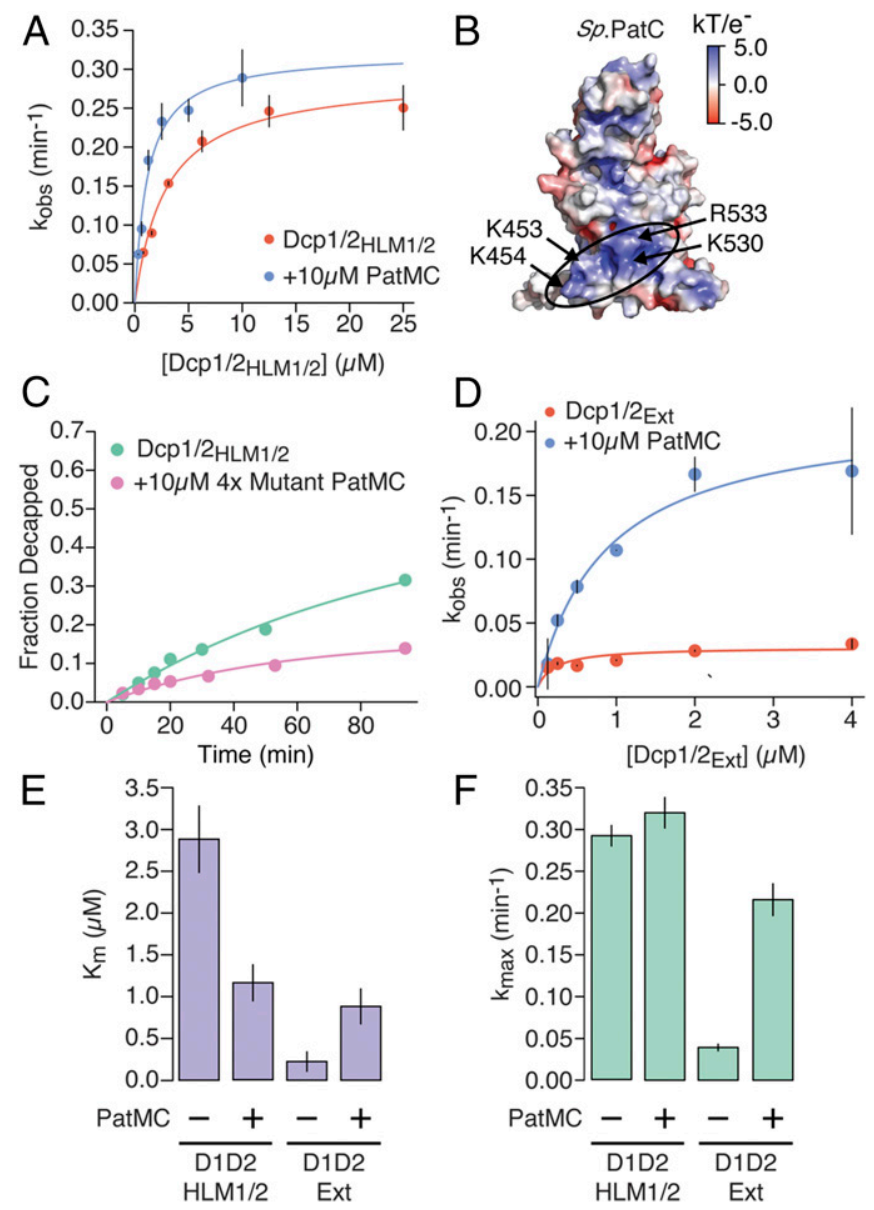

Fig. 5. The middle domain differentially activates C-terminally extended Dcp2 constructs. (A) Single-turnover kinetic analyses of Dcp1/Dcp2 $2_{\mathrm{HLM} 1 / 2}$ alone or in the presence of $10 \mu \mathrm{M}$ MBP-PatMC $(n=2)$. (B) Electrostatic surface representation of a model of SpPatC based on PDB 5LMG using MODELLER (45) and APBS in PyMOL (Schrödinger). The basic patch is circled, while residues mutated are indicated with arrows. (C) Representative decapping assay of a RNA with $150 \mathrm{nM}$ Dcp1/Dcp2 $2_{\mathrm{HLM} 1 / 2}$ and $10 \mu \mathrm{M} 4 \times$ mutant MBP-PatMC (K453A K454A/K530A/R533A). (D) Single-turnover kinetic analyses of autoinhibited Dcp1/Dcp2 $2_{\text {Ext }}$ alone or in the presence of $10 \mu \mathrm{M} \mathrm{MBP-PatMC}(n=2)$. ( $E$ and $\left.F\right)$ $\mathrm{K}_{\mathrm{M}}$ and $\mathrm{k}_{\max }$ values calculated from $A$ and $D$ for Dcp1/Dcp2 ${ }_{\mathrm{HLM} 1 / 2}$ or Dcp1/ $\mathrm{DCp}_{\mathrm{Ext}}$ alone or in the presence of $10 \mu \mathrm{M}$ MBP-PatMC shown with SD. 
while also decreasing the affinity of Dcp1/Dcp2 ${ }_{\text {Ext }}$ for the substrate (Fig. $5 A, C$, and $D$ ). This suggests that Dcp1/Dcp2 ${ }_{\text {Ext }}$ binds nonproductively to RNA and PatMC activates catalysis at the expense of substrate binding. This is in agreement with HLM-binding proteins relieving autoinhibition to promote decapping, suggesting this may be a general model for proteins that bind the $\mathrm{C}$ terminus of Dcp2. We conclude Pat1 uses its middle and C-terminal domains to alleviate autoinhibition and enhance substrate binding of the Dcp1/Dcp2 decapping enzyme complex.

\section{Discussion}

Pat1 works together with Dcp1/Dcp2 and the Lsm1-7 complex to promote bulk 5'-3' decay on numerous transcripts in yeast (12). Our work reveals how Pat1 interacts with and activates separate steps in this pathway. First, Pat1 enhances the RNA binding of the Lsm1-7 complex using a bipartite interaction surface comprised of its middle and C-terminal domains. Second, Pat1 promotes decapping by Dcp1/Dcp2 using both domains to affect RNA binding and alleviate autoinhibition. Taken together, these results provide a mechanistic framework for how Pat1 can enhance binding and activity of factors that act on the $5^{\prime}$ and $3^{\prime}$ ends of mRNA to promote bulk decay (Fig. 6).

Pat1 enhances binding of the Lsm1-7 complex to oligoA RNA 10 -fold using bipartite interactions of its middle and C-terminal domains (Fig. 1). This requires short-linear motifs within the middle domain of Pat1, termed the LBM and the LAR, that mediate the complex formation with Lsm1-7 and enhance oligoA binding (Figs. 2 and 3). Interactions of the C-terminal domain of Pat 1 with Lsm $2 / 3$ as predicted by crystallographic analyses are necessary for the assembly of a stable complex among Pat1, the Lsm1-7 ring, and RNA. Pat1 constructs lacking the LAR still interact with Lsm1-7 but fail to enhance RNA binding. Therefore, protein interactions required for the formation of a stable Pat 1 and Lsm1-7 complex can be separated from the ability of Pat1 to enhance RNA binding of Lsm1-7. The middle domain plays a role beyond promoting the interaction between Pat1 and Lsm1-7 and is likely involved in stabilizing a conformation of Lsm1-7 that can bind RNA with high affinity. The molecular details of how the middle domain of Pat1 interacts with the Lsm1-7 ring and enhances RNA binding will require high-resolution structural studies.

The mechanism by which the Pat1/Lsm1-7 complex recognizes and targets mRNAs for decapping remains poorly understood. In budding yeast, Pat1/Lsm1-7 binds the $3^{\prime}$ end of mRNAs and promotes degradation of inefficiently translated transcripts.

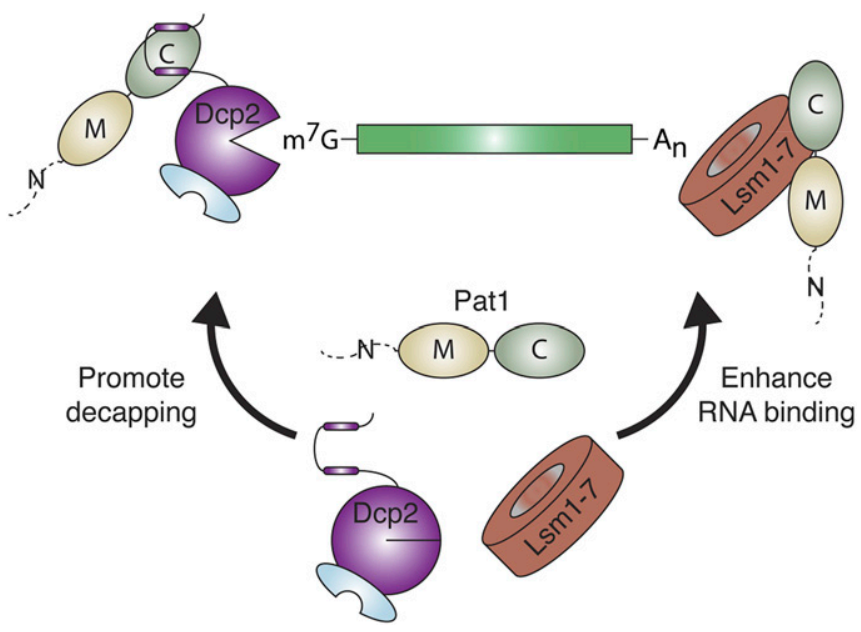

Fig. 6. Model for how Pat1 interacts with and activates multiple RNA decay factors to establish $5^{\prime}$ decapping. Pat 1 interacts with and activates decay factors at both the $5^{\prime}$ and the $3^{\prime}$ ends of the mRNA to promote decapping.
Furthermore, biochemical evidence suggests preferential binding of deadenylated oligoA tails $(12,29,30)$. In $S p$ and higher order eukaryotes, however, an additional 1-3 uridines are added to a subset of transcripts after deadenylation but prior to decapping, which are enriched when $\operatorname{Lsm} 1$ is deleted $(19,20)$. Understanding how different mRNA tail modifications affect Pat1/ Lsm1-7 recognition in cells remains a challenge for the future.

Multiple decapping activators bind the $\mathrm{C}$-terminal regulatory region of Dcp2 to activate decapping and ensure transcript specificity in cells. We show that Pat1 directly activates decapping by binding HLMs in the C-terminal regulatory region to enhance Dcp1/Dcp2 RNA binding and alleviate autoinhibition (Figs. 4 and 5). This mechanism is reminiscent of other HLM-binding proteins, such as Edc3, and may be a general mechanism by which HLM-binding proteins promote decapping. Importantly, the middle domain and structured $\mathrm{C}$ terminus are both required for these effects. This is consistent with early work showing the middle domain is required to activate decapping in yeast and recent structural work showing the $\mathrm{C}$-terminal domain binds Dcp2 $(16,41)$. Taken with our biochemical characterization of the Pat1/Lsm1-7 interaction, these observations suggest that both the middle and the C-terminal domains of Pat1 are required to activate distinct steps of mRNA decay. It is likely that deletion of the middle domain has a strong defect in decapping because of reduced Lsm1-7 RNA binding and activity of Dcp1/Dcp2 on substrate mRNA.

Previous studies from budding yeast, flies, and humans have demonstrated that Pat1 interacts with multiple decay factors and is required for normal mRNA turnover in vivo, which requires both the unstructured middle domain and the globular C-terminal domain. In humans, PatC can interact with Lsm1-7, while in flies, the middle domain is sufficient for association (38, 40). Budding yeast PatC can interact with Lsm1-7 but requires cooperation between both the middle and the C-terminal domains to promote RNA binding $(27,28,37)$. Both the middle and the C-terminal domains in flies can interact with Dcp2, although in humans and budding yeast, the C-terminal domain of Pat1 is sufficient $(16,38,40,41,43)$. It is clear that the relative strengths of the interactions between Pat1 and different RNA decay factors have changed during the course of evolution, which may be a potential mode of regulation for the assembly of RNA decay factors. Our biochemically reconstituted system defines how short-linear motifs in the disordered middle domain with the structured $\mathrm{C}$-terminal domain cooperate to promote distinct steps during $5^{\prime}-3^{\prime}$ mRNA degradation.

Our work demonstrates how conserved short-linear motifs of the unstructured middle domain of SpPat1 enhance Lsm1-7, which may be applicable to nuclear Lsm2-8 complexes. Recent genome-wide analysis of the vertebrate ortholog of Pat1 (Pat1b/ PatL1) suggest it functions with the Lsm2-8 complex during premRNA splicing in addition to its established role with Lsm1-7 in mRNA decay. Ablation of Pat1b promotes skipping of exons with weak donor site sequences in addition to increasing the steady-state levels of AU rich containing mRNAs. Knockdown of Pat1b reduces formation of the U6 snRNP containing Lsm2-8 and SART3 (an ortholog of yeast Prp24). Furthermore, Pat1b is localized in nuclear Cajal bodies as well as in cytoplasmic processing bodies (44). The unstructured middle domain of Pat1 may endow it with structural plasticity, allowing it to function as a versatile chaperone for RNP assembly during bulk $5^{\prime}-3^{\prime}$ mRNA decay and pre-mRNA splicing.

\section{Materials and Methods}

Details about methods including protein purification, NMR spectroscopy analytical size exclusion chromatography, RNA transcription and capping, decapping kinetics, fluorescence polarization, pull-downs, and yeast strain construction can be found in the SI Appendix, Materials and Methods. Sp strains used in this study are in SI Appendix, Table S1. 
Data Availability. Plots and fits were generated using in house scripts written in $\mathrm{R}$, which are available at https://github.com/jhlobel/FP-fitting.

ACKNOWLEDGMENTS. We thank R. A. Greenstein and Sandra Catania for help with the construction of yeast strains, members of the J.D.G. Laboratory

1. R. Parker, RNA degradation in Saccharomyces cerevisae. Genetics 191, 671-702 (2012).

2. M. J. Moore, From birth to death: The complex lives of eukaryotic mRNAs. Science 309, 1514-1518 (2005)

3. A. Stevens, Purification and characterization of a Saccharomyces cerevisiae exoribonuclease which yields $5^{\prime}$-mononucleotides by a $5^{\prime}$ leads to $3^{\prime}$ mode of hydrolysis. J. Biol. Chem 255, 3080-3085 (1980).

4. H. Guo, N. T. Ingolia, J. S. Weissman, D. P. Bartel, Mammalian microRNAs pre dominantly act to decrease target mRNA levels. Nature 466, 835-840 (2010).

5. S. Jonas, E. Izaurralde, Towards a molecular understanding of microRNA-mediated gene silencing. Nat. Rev. Genet. 16, 421-433 (2015).

6. T. M. Franks, J. Lykke-Andersen, The control of mRNA decapping and P-body formation Mol. Cell 32, 605-615 (2008).

7. S. Kervestin, A. Jacobson, NMD: A multifaceted response to premature translational termination. Nat. Rev. Mol. Cell Biol. 13, 700-712 (2012).

8. E. van Dijk et al., Human Dcp2: A catalytically active mRNA decapping enzyme located in specific cytoplasmic structures. EMBO J. 21, 6915-6924 (2002).

9. T. Dunckley, R. Parker, The DCP2 protein is required for mRNA decapping in Saccharomyces cerevisiae and contains a functional MutT motif. EMBO J. 18, 5411-5422 (1999).

10. G. Badis, C. Saveanu, M. Fromont-Racine, A. Jacquier, Targeted mRNA degradation by deadenylation-independent decapping. Mol. Cell 15, 5-15 (2004).

11. S. Dong et al., YRA1 autoregulation requires nuclear export and cytoplasmic Edc3pmediated degradation of its pre-mRNA. Mol. Cell 25, 559-573 (2007).

12. F. He, A. Celik, C. Wu, A. Jacobson, General decapping activators target different subsets of inefficiently translated mRNAs. elife 7, e34409 (2018).

13. Z. Wang, X. Jiao, A. Carr-Schmid, M. Kiledjian, The hDcp2 protein is a mammalian mRNA decapping enzyme. Proc. Natl. Acad. Sci. U.S.A. 99, 12663-12668 (2002).

14. J. S. Mugridge, R. W. Tibble, M. Ziemniak, J. Jemielity, J. D. Gross, Structure of the activated Edc1-Dcp1-Dcp2-Edc3 mRNA decapping complex with substrate analog poised for catalysis. Nat. Commun. 9, 1152 (2018).

15. S. A. Fromm et al., The structural basis of Edc3- and Scd6-mediated activation of the Dcp1:Dcp2 mRNA decapping complex. EMBO J. 31, 279-290 (2012).

16. $\mathrm{F}$. $\mathrm{He}, \mathrm{A}$. Jacobson, Control of mRNA decapping by positive and negative regulatory elements in the Dcp2 C-terminal domain. RNA 21, 1633-1647 (2015).

17. D. R. Paquette, R. W. Tibble, T. S. Daifuku, J. D. Gross, Control of mRNA decapping by autoinhibition. Nucleic Acids Res. 46, 6318-6329 (2018).

18. S. Jonas, E. Izaurralde, The role of disordered protein regions in the assembly of decapping complexes and RNP granules. Genes Dev. 27, 2628-2641 (2013).

19. O. S. Rissland, C. J. Norbury, Decapping is preceded by $3^{\prime}$ uridylation in a novel pathway of bulk mRNA turnover. Nat. Struct. Mol. Biol. 16, 616-623 (2009).

20. J. Lim et al., Uridylation by TUT4 and TUT7 marks mRNA for degradation. Cell 159 1365-1376 (2014)

21. S. Tharun, R. Parker, Targeting an mRNA for decapping: Displacement of translation factors and association of the Lsm $1 \mathrm{p}-7 \mathrm{p}$ complex on deadenylated yeast mRNAs. Mol. Cell 8, 1075-1083 (2001).

22. S. Tharun et al., Yeast Sm-like proteins function in mRNA decapping and decay. Nature 404, 515-518 (2000).

23. E. Bouveret, G. Rigaut, A. Shevchenko, M. Wilm, B. Séraphin, A Sm-like protein complex that participates in mRNA degradation. EMBO J. 19, 1661-1671 (2000). for discussions, and Jeffrey S. Mugridge and Krister J. Barkovich for comments on the paper. R.W.T. was supported by a Genentech Foundation predoctoral fellowship and UCSF discovery fellowship. This work was supported by US National Institutes of Health Grant R01 GM078360 (to J.D.G.).

24. W. He, R. Parker, The yeast cytoplasmic Lsml/Pat1p complex protects mRNA 3' termini from partial degradation. Genetics 158, 1445-1455 (2001).

25. S. Tharun, D. Muhlrad, A. Chowdhury, R. Parker, Mutations in the Saccharomyces cerevisiae LSM1 gene that affect mRNA decapping and 3' end protection. Genetics 170, 33-46 (2005).

26. C. Bonnerot, R. Boeck, B. Lapeyre, The two proteins Pat1p (Mrt1p) and Spb8p interact in vivo, are required for mRNA decay, and are functionally linked to Pab1p. Mol. Cell. Biol. 20, 5939-5946 (2000).

27. H. Sharif, E. Conti, Architecture of the Lsm1-7-Pat1 complex: A conserved assembly in eukaryotic mRNA turnover. Cell Rep. 5, 283-291 (2013).

28. D. Wu et al., Lsm2 and Lsm3 bridge the interaction of the Lsm1-7 complex with Pat1 for decapping activation. Cell Res. 24, 233-246 (2014).

29. A. Chowdhury, J. Mukhopadhyay, S. Tharun, The decapping activator Lsm1p-7p-Pat1p complex has the intrinsic ability to distinguish between oligoadenylated and polyadenylated RNAs. RNA 13, 998-1016 (2007).

30. S. F. Mitchell, S. Jain, M. She, R. Parker, Global analysis of yeast mRNPs. Nat. Struct. Mol. Biol. 20, 127-133 (2013)

31. C.-Y. Wang, Y.-T. Wang, W.-Y. Hsiao, S.-W. Wang, Involvement of fission yeast Pdc2 in RNA degradation and P-body function. RNA 23, 493-503 (2017).

32. J.-Y. Youn et al., High-density proximity mapping reveals the subcellular organization of mRNA-associated granules and bodies. Mol. Cell 69, 517-532.e11 (2018).

33. A. Hubstenberger et al., P-body purification reveals the condensation of repressed mRNA regulons. Mol. Cell 68, 144-157.e5 (2017).

34. D. Teixeira, R. Parker, Analysis of P-body assembly in Saccharomyces cerevisiae. Mol. Biol. Cell 18, 2274-2287 (2007).

35. H. Sharif et al., Structural analysis of the yeast Dhh1-Pat1 complex reveals how Dhh1 engages Pat1, Edc3 and RNA in mutually exclusive interactions. Nucleic Acids Res. 41, 8377-8390 (2013).

36. G. R. Pilkington, R. Parker, Pat1 contains distinct functional domains that promote P-body assembly and activation of decapping. Mol. Cell. Biol. 28, 1298-1312 (2008).

37. A. Chowdhury, S. Kalurupalle, S. Tharun, Pat1 contributes to the RNA binding activity of the Lsm1-7-Pat1 complex. RNA 20, 1465-1475 (2014).

38. J. E. Braun et al., The C-terminal $\alpha-\alpha$ superhelix of Pat is required for mRNA decapping in metazoa. EMBO J. 29, 2368-2380 (2010).

39. Z. Fourati et al., The C-terminal domain from S. cerevisiae Pat1 displays two conserved regions involved in decapping factor recruitment. PLoS One 9, e96828 (2014).

40. G. Haas et al., HPat provides a link between deadenylation and decapping in metazoa. J. Cell Biol. 189, 289-302 (2010)

41. C. Charenton et al., A unique surface on Pat1 C-terminal domain directly interacts with Dcp2 decapping enzyme and Xrn1 5'-3' mRNA exonuclease in yeast. Proc. Natl. Acad. Sci. U.S.A. 114, E9493-E9501 (2017).

42. J. P. Wurm, J. Overbeck, R. Sprangers, The $S$. pombe mRNA decapping complex recruits cofactors and an Edc1-like activator through a single dynamic surface. RNA 22, 1360-1372 (2016)

43. T. Nissan, P. Rajyaguru, M. She, H. Song, R. Parker, Decapping activators in Saccharomyces cerevisiae act by multiple mechanisms. Mol. Cell 39, 773-783 (2010).

44. C. Vindry et al., Dual RNA processing roles of Pat $1 \mathrm{~b}$ via cytoplasmic Lsm1-7 and nuclear Lsm2-8 complexes. Cell Rep. 20, 1187-1200 (2017).

45. N. Eswar et al., Comparative protein structure modeling using modeller. Curr. Protoc. Bioinformatics, 15, 5.6.1-5.6.30 (2014). 